\title{
МИЕЛОМНАЯ НЕФРОПАТИЯ
}

\author{
Зудерман Н.Е. ${ }^{1}$, Ушакова Н.Д. ${ }^{1}$, Лысенко И.Б. ${ }^{\text {, Тен И.А. }}{ }^{1}$ \\ ${ }^{1}$ ФББУ «Национальный медицинский исследовательский иеетр онкологии» Министерства здравоохранения \\ Российской Федерации, Ростов-на-Дону, е-mail: natalka8n@yandex.ru
}

Работа посвящена возможности применения в комплексе сопроводительного лечения больных секретирующей множественной миеломой экстракорпоральной детоксикации. Известно, что повреждение почек в дебюте множественной миеломы наблюдается у $50 \%$ больных, из них в $10 \%$ случаев почечная недостаточность требует проведения заместительной почечной терапии. Нарушение функции почек ассоциировано с развитием осложнений, ухудшением качества жизни. Почечная недостаточность занимает второе место среди причин смерти при множественной миеломе. Больные множественной миеломой составляют около $2 \%$ в популяции диализных больных, и риск смерти у них в 2,8 раза выше. Традиционно при лечении тяжелой почечной недостаточности у больных множественной миеломой применяют гемодиализ. Тем не менее при прогрессирующей множественной миеломе и отсутствии гематологического ответа на химиотерапию около $20 \%$ больных умирают в течение первого месяца, а в $50 \%$ случаев продолжительность их жизни не превышает года. Учитывая низкую частоту почечного ответа у больных множественной миеломой и диализзависимой почечной недостаточностью продолжается поиск вариантов лечения данной категории больных с применением химиотерапии в сочетании с диализным лечением и экстракорпоральным удалением свободных легких цепей иммуноглобулинов.

Ключевые слова: миеломная нефропатия, свободные легкие цепи иммуноглобулинов, экстракорпоральная детоксикация.

\section{MYELOMA NEPHROPATHY}

\author{
Zuderman N.E. ${ }^{1}$, Ushakova N.D. ${ }^{1}$, Lysenko I.B. ${ }^{1}$, Ten I.A. ${ }^{1}$

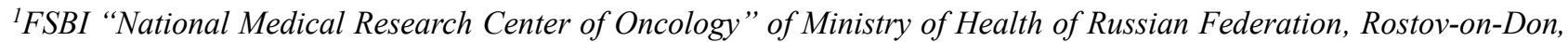 \\ e-mail:natalka8n@yandex.ru
}

This article is devoted to the possible use of extracorporeal detoxification in the complex of accompanying treatment in patients with secretory multiple myeloma. Kidney damage in the onset of multiple myeloma is observed in $50 \%$ of patients, including $10 \%$ of renal failure cases requiring renal replacement therapy. Renal dysfunction is associated with the development of complications and a poor quality of life. Renal failure is the second-leading cause of death in multiple myeloma. Patients with multiple myeloma amount for about $2 \%$ of the dialysis patient population, and the risk of death in them is $\mathbf{2 . 8}$ times higher. Hemodialysis is traditionally used in the treatment of acute renal failure in patients with multiple myeloma. Nevertheless, about $20 \%$ of patients with progressive multiple myeloma without hematological response to chemotherapy die within the first month, and in $\mathbf{5 0 \%}$ of cases the survival does not exceed a year. Low rates of renal response in patients with multiple myeloma and dialysis-dependent renal failure require the further search for treatment options for this category of patients using chemotherapy in combination with dialysis treatment and extracorporeal removal of immunoglobulin free light chains.

Keywords: myeloma nephropathy, immunoglobulin free light chains, extracorporeal detoxification.

Множественная миелома (MM) - злокачественное лимфопролиферативное заболевание, которое характеризуется пролиферацией клональных плазматических клеток как следствие хромосомных нарушений и стромального микроокружения, продукцией моноклонального белка [1]. ММ - один из самых распространенных гемобластозов. Множественная миелома составляет 1\% от онкологических заболеваний, а гематологических опухолей - около $12 \%$.

Этиология множественной миеломы в настоящее время не определена. Возможными 
мутагенов (асбест, производные бензола, инсектициды), употребление алкоголя, длительную антигенную стимуляцию на фоне хронических воспалительных заболеваний. Ожирение также является предрасполагающим фактором развития заболевания. Так, увеличение индекса массы тела на 5 единиц увеличивает риск развития заболевания на $11 \%$. Определенное значение в этиологии ММ отводят генетическим факторам и наследственной предрасположенности, что косвенно подтверждается более частым развитием ММ у близких родственников и однояйцевых близнецов [1;2]. По мнению A.K. Dutta et al. (2017), тлеющая миелома также увеличивает риск развития онкологического процесса [3]. В исследованиях О. Landgren et al. (2009) и Willrich M.A. et al. (2017) показано, что почти во всех случаях развитию множественной миеломы предшествовала моноклональная гаммопатия неопределенного генеза (MGUS) [4; 5].

В основе патогенеза ММ лежит опухолевая трансформация клеток-предшественниц В-лимфоцитов в терминальном центре периферических лимфоидных органов после соматических гипермутаций реаранжированных генов иммуноглобулинов и изотипического переключения синтеза антител с сохранением способности этих клеток дифференцироваться до конечного этапа - плазматических клеток, секретирующих М-белок. М-белок - это иммуноглобулин (Ig) или его компонент/фрагмент с аномальной последовательностью в своем строении аминокислот и потерей нормальной функции в результате мутации генов, ответственных за выработку иммуноглобулина [6].

Клиническая картина ММ разнообразна и обусловлена основными патогенетическими признаками заболевания: инфильтрацией костного мозга клональными плазматическими клетками; секрецией патологического моноклонального иммуноглобулина; существенным угнетением продукции нормальных поликлональных иммуноглобулинов. Клинические проявления при ММ включают костномозговой синдром (угнетение нормального гемопоэза как следствие плазмоклеточной инфильтрации костного мозга, поражение костей вследствие остеодеструкции); гиперкальциемию; нарушение функции почек; синдром повышенной вязкости крови; амилоидоз; геморрагический синдром; неврологические нарушения; синдром вторичного иммунодефицита [7]. Как правило, клинически ММ проявляется, когда масса опухоли составляет около $10^{12}$ клеток [1].

Неконтролируемый рост плазматических клеток приводит к угнетению нормального кроветворения в костном мозге с развитием анемии, нейтропении, реже тромбоцитопении. При первичной диагностике ММ снижение уровня гемоглобина менее 100 г/л выявляется у 60-70\% больных. Нейтропения и тромбоцитопения чаще всего наблюдаются при поздних стадиях заболевания и сопровождаются повышением СОЭ. 
Диагностические критерии множественной миеломы основаны на данных клиниколабораторного и инструментального обследования. Согласно национальным рекомендациям по диагностике и лечению множественной миеломы обследование больных ММ включает: общеклиническое обследование; определения статуса по ECOG; определение сывороточного содержания $\beta_{2}$-микроглобулина, свободных легких цепей $\mathrm{Ig} ;$ электрофорез и иммунофиксацию белков сыворотки крови и суточной мочи с количественным определением М-градиента в крови и моноклонального белка в моче; иммунологическое обследование; морфологическое исследование аспирата костного мозга и/или трепанобиоптата, лучевую диагностику. Состояние функции почек оценивают по скорости клубочковой фильтрации (СКФ), которую определяют расчетными методами по формуле MDRD (Modification of Diet in Renal Disease) или Cockcroft-Gault.

Успех лечения множественной миеломы зависит как от выбора оптимального метода противоопухолевой терапии, так и от эффективности борьбы с многочисленными осложнениями, сопровождающими развитие заболевания [6; 8]. Эндогенная интоксикация и почечная недостаточность являются наиболее грозными осложнениями ММ, закономерно ухудшающими состояние больного при ММ и значительно затрудняющими проведение адекватной химиотерапии, что, соответственно, ухудшает прогноз и исход заболевания [9]. В связи с этим сопроводительная терапия является неотъемлемой частью лечения больных с гемобластозами.

Цель исследования: провести анализ данных современной литературы и принципов лечения миеломной нефропатии.

Повреждение почек в дебюте множественной миеломы (MМ) наблюдается у $50 \%$ больных. При этом 20\% имеют почечную недостаточность, которая в $10 \%$ случаев требует проведения заместительной почечной терапии. У 25\% больных почечная недостаточность развивается на фоне специфического лечения при рецидивирующей и рефрактерной миеломе [10]. По данным международных эпидемиологических исследований отмечается рост почечной патологии, связанной с множественной миеломой от 0,7 млн случаев в 1986-1990 годах до 6-8 млн в 2010 году. Нарушение функции почек ассоциировано с развитием осложнений, ухудшением качества жизни. Почечная недостаточность занимает второе место среди причин смерти при множественной миеломе. Больные множественной миеломой составляют около $2 \%$ в популяции диализных больных, и риск смерти у них в 2,8 раза выше [9; 11-13]. Наиболее часто при множественной миеломе отмечают развитие миеломной тубулярной нефропатии - в 6080\% случаев, в 10-20\% случаев выявляют AL-амилоидоз, у 5-10\% больных диагностируют болезнь депозитов легких цепей. К редким вариантам почечного повреждения относят 

хронический тубулоинтерстициальный нефрит, плазмоклеточную инфильтрацию [14].

В настоящее время в патогенезе почечных нарушений при множественной миеломе условно выделяют три основных механизма [15; 16].

Первый обусловлен нефротоксическим воздействием циркулирующего в сыворотке и экскретируемого с мочой парапротеина. Клетки, секретирующие иммуноглобулины, продуцируют легкие цепи, которые в большом количестве фильтруются через канальцы почек. Реабсорбция легких цепей иммуноглобулинов приводит к повреждению эпителия канальцев и нарушению их функции. Патогенез миеломной нефропатии зависит от локализации повреждения почечных канальцев. При ММ патологические изменения могут затрагивать практически все отделы нефрона - тубулоинтерстициальные поражения различной степени выраженности и AL-амилоидоз.

Преципитация свободных легких цепей в дистальных отделах почечных канальцев с белком Тамма-Хорсфалла приводит к развитию интерстициального воспаления и обструкции канальцев, тубулорексису. При морфологическом исследовании нефробиоптата выявляются атрофия канальцев, интерстициальное воспаление, фиброз. Повреждение проксимальных канальцев сопровождается выраженным эндоцитозом. Свободные легкие цепи иммуноглобулинов (СЛЦ Ig) активируют редокс-сигнальные пути апоптоза, увеличивают экспрессию NF-кB, индуцируя синтез провоспалительных цитокинов. Морфологическая картина характеризуется тубулонекрозом, воспалением, фиброзом [17].

Третий механизм составляют системные проявления множественной миеломы гиперкальциемия, гиперурикемия, повышенная вязкость крови, гиповолемия, анемия, рецидивирующая инфекция и др. Моноклональные СЛЦ Ig способствуют быстрому склерозированию, и через 1,5-2 месяца формируется интерстициальный фиброз и ХПН [18; 19]. Нарушение функции почек ухудшает клинические результаты специфического лечения и сокращает продолжительность жизни. Медиана выживаемости больных при диализзависимой почечной недостаточности составляет 10,2 месяца. В связи с этим целью лечения больных $\mathrm{MM}$ и миеломной нефропатией является достижение как гематологического, так и почечного ответа [6;12].

Сопроводительное лечение на ранних стадиях миеломной нефропатии включает в себя: коррекцию дегидратации солевыми, щелочными растворами не менее 3 литров в сутки; поддержание РН мочи $\geq 7,0$; коррекцию гиперкальциемии (регидратация, исключение петлевых диуретиков, назначение аллопуринола), бисфосфонаты с коррекцией дозы соответственно СКФ; исключение нестероидных противовоспалительных препаратов, блокаторов ренин-альдестероновой системы, нефротоксичных антибиотиков. 
При гипервискозном синдроме проводят лечебный плазмаферез. В случаях развития поздних стадий почечной недостаточности в комплекс терапии включают диализное лечение, которое проводят по общепринятым показаниям (СКФ менее 10 мл/мин., некорригируемые нарушения водно-электролитного баланса и кислотно-щелочного состояния).

Индукционную терапию проводят высокими дозами стероидов (HD-Dex) 40 мг/сутки в течение 4 дней в сочетании с препаратами, не требующими коррекции дозы при сниженной СКФ: циклофосфамид, талидомид, бортезомиб, бендамустин, адриамицин. Исключают препараты с почечной элиминацией: мелфалан, леналидомид. Прогресс последних десятилетий в лечении ММ связан с применением в клинической практике новых цитостатиков - бортезомиба и леналидомида, а также с использованием аутотрансплантации гемопоэтических стволовых клеток [20-23].

Бортезомиб является одним из препаратов, рекомендованных для инициального лечения. Он не обладает миелосупрессивным воздействием и обеспечивает быстрый гематологический ответ [24; 25]. В настоящее время бортезомиб включен в программы VCD (бортезомиб, циклофосфамид, дексаметазон) или PAD (бортезомиб, адриабластин, дексаметазон) - терапию первой линии при миеломной нефропатии.

При достижении хорошего гематологического ответа больным до 65 лет при отсутствии тяжелых сопутствующих заболеваний выполняют аутотрансплантацию стволовых клеток. При этом почечная недостаточность, в том числе и диализзависимая, не является противопоказанием к ее проведению. При достижении после 2-4 курсов химиотерапии частичной ремиссии и сохранении почечной недостаточности предпочтителен переход на вторую линию: схемы с талидомидом, ленолидомидом ( $\mathrm{Rd}$ - ленолидомид + Dex), бендамустином (ВР - бендамустин + преднизолон).

Больным MM старше 70 лет индукционную терапию проводят по программе VCD c возрастной коррекцией доз. При тяжелой нефропатии и СКФ менее 30 мл/мин. и удовлетворительном соматическом состоянии возможно проведение 1-2 индукционных курсов VCD с введением полных доз бортезомиба и циклофосфамида два раза в неделю при снижении дозы дексаметазона до 20-40 мг в неделю. После достижения почечного ответа необходимо редуцировать дозы соответственно возрасту.

При лечении критических состояний, коррекции эндогенной интоксикации, в том числе и при почечной недостаточности различной этиологии, все более широко применяют методы экстракорпоральной детоксикации - удаление токсических веществ из организма в неизменном виде [26]. 
Согласно российским клиническим рекомендациям по диагностике и лечению миеломной нефропатии (2014) стандартом лечения этой категории больных является химиотерапия и гидратация [27]. При диагностике ММ и миеломной нефропатии необходимо незамедлительное начало химиотерапевтического лечения, поскольку восстановление функции почек возможно только в условиях редукции опухоли.

Традиционно при лечении тяжелой почечной недостаточности у больных ММ применяют гемодиализ. Тем не менее при прогрессирующей $\mathrm{MM}$ и отсутствии гематологического ответа на химиотерапию около $20 \%$ больных умирают в течение первого месяца, а в 50\% случаев продолжительность их жизни не превышает года. По данным финского медицинского общества (2007), в странах Европы продолжительность жизни у диализзависимых больных миеломной нефропатией составляет 3,5 месяца [28]. Тем не менее диализное лечение остается перспективным в лечении данной категории больных, поскольку улучшение функции почек на фоне специфической терапии может задерживаться на несколько месяцев [29]. Кроме того, следует учитывать, что более раннее использование диализного лечения может позволить избежать критической уремии, значительно осложняющей течение основного заболевания. Л.С. Бирюкова с соавт. (2002) считают показанием к началу гемодиализа при $\mathrm{MM}$ концентрации креатинина крови 500600 мкмоль/л (из-за дефицита массы тела у 40\% больных миеломной нефропатией) [30]. В настоящее время в литературе имеются лишь единичные данные о восстановлении функции почек у больных, находившихся на гемодиализе и получающих полихимиотерапию [31].

Учитывая низкую частоту почечного ответа у больных ММ и диализзависимой почечной недостаточностью продолжается поиск вариантов лечения данной категории больных с применением разных схем химиотерапии в сочетании с диализным лечением и экстракорпоральным удалением СЛЦ Ig. Исследования в этом направлении продолжаются. C.A. Hutchison et al. (2009) показали, что проведение продленного гемодиализа с использованием гемофильтров с высокопроницаемой для белков мембраны НСОHD площадью 1,1 м² у 67 больных миеломной нефропатией обеспечивало через 2 часа от начала процедуры эффективное удаление СЛЦ Ig в среднем на 30-70\% и достижение высокого уровня независимости от гемодиализа после завершения специфического лечения около $60 \%$ [32]. В настоящее время завершено Европейское рандомизированное исследование по эффективности почечного ответа у больных ММ и диализзависимой почечной недостаточностью. В исследование было включено 90 больных, 45 из которых диализное лечения проводили с использованием обычных мембран диализатора, 45 высокопроницаемых. Статистически значимых отличий в восстановлении функции почек в течение 3 месяцев в группах исследования выявлено не было [33]. 
Предварительные данные другого мультицентрового рандомизированного исследования подтверждают высокую частоту обратимости почечной недостаточности при применении в комплексе противоопухолевого лечения больных множественной миеломой гемодиализа с использованием высокопроницаемых для СЛЦ фильтров [34]. Следует отметить, что в это исследование были включены только больные при гистологически доказанной cast-нефропатии, a химиотерапию проводили по бортезомибсодержащим протоколам. Крайне важным является и тот факт, что улучшение функции почек наблюдали лишь у больных, у которых был получен гематологический ответ.

На целесообразность экстракорпорального удаления СЛЦ Ig в процессе проведения диализного лечения больных ММ указывают в своих исследованиях И.Г. Рехтина с соавт. $(2013,2015)[20 ; 35]$.

Представленные выше данные касаются лечения уже состоявшейся при ММ почечной недостаточности, требующей заместительной почечной терапии. Н.А. Любимова с соавт. (2017), Е.Г. Громова с соавт. (2019), применив экстракорпоральную гемокоррекцию с использованием селективных высокопроницаемых полисульфоновых фильтров EMic2 с площадью поверхности мембраны $1,8 \mathrm{~m}^{2}$ и способностью, благодаря размеру пор, фильтрации и удаления веществ с молекулярной массой до 60000 дальтон у больных моноклональными гаммапатиями с высокой продукций СЛЦ, высказывают мнение о целесообразности экстракорпорального удаления из кровотока избытка СЛЦ вне зависимости от наличия и стадии почечной дисфункции [36; 37].

\section{Заключение}

Развитие диализзависимой почечной недостаточности у больных ММ является «медицинской катастрофой», для предупреждения ее развития у больных секретирующей ММ представляется перспективным использование различных вариантов сопроводительного лечения с включением экстракорпоральных методов лечения.

\section{Список литературы}

1. Богданов А.Н., Мазуров В.И. Клиническая гематология. Руководство для врачей. СПб., 2008. 484 с.

2. Roberts D.L., Dive C., Renehan A.G. Biological mechanisms linking obesity and cancer risk: new perspectives. Annual Review of Medicine. 2010. no. 61. P. 301-16.

3. Dutta A.K., Hewett D.R., Fink J.L. Cutting edge genomics reveal new insights into tumour development, disease progression and therapeutic impacts in multiple myeloma. British Journal of 
Haematology. 2017. vol. 178 no. 2. P. 196-208. DOI: 10.1111/bjh.14649.

4. Landgren O., Kyle R.A., Pfeiffer R.M., Katzmann J.A., Caporaso N.E., Hayes R.B., Dispenzieri A., Kumar S., Clark R.J., Baris D., Hoover R., Rajkumar S.V. Monoclonal gammopathy of undetermined significance (MGUS) consistently precedes multiple myeloma: a prospective study. Blood. 2009. vol. 113. no. 22. P. 5412-7. DOI: 10.1182/blood-2008-12-194241. 5. Willrich M.A., Murray D.L., Kyle R.A. Laboratory testing for monoclonal gammopathies: Focus on monoclonal gammopathy of undetermined significance and smoldering multiple myeloma. Clinical Biochemistry. 2017. no. 51. P.38-47. DOI: 10.1016/j.clinbiochem.2017.05.001.

6. Бессмельцев, С.С., Абдулкадыров К.М. Множественная миелома: руководство для врачей. М.: Специальное изд-во мед. кн., 2016. 493 с.

7. равченко Д.В., Ходулева С.А., Новик Д.К. Множественная миелома. Гомель: ГУ «РНПЦ и ЭЧ» Практическое пособие для врачей, 2016. 83 с.

8. Kumar S.K., Rajkumar S.V., Dispenzieri A. Lacy M.Q., Hayman S.R., Buadi F.K., Zeldenrust S.R., Dingli D., Russell S.J., Lust J.A., Greipp P.R., Kyle R.A., Gertz M.A. Improved survival in multiple myeloma and the impact of novel therapies. Blood. 2008. vol. 111. no. 5. P. $2516-2520$.

9. Karin M., Cao Y., Greten F.R., Li Z.W. NF-kappa B in cancer: from innocent bystander to major culprit. Nat. Rev. Cancer. 2002. no. 2. P. 301-310.

10. Wirk B. Renal failure in multiple myeloma: a medical emergency Bone Marrow Transplantation. 2011. no. 46. P. 771-783.

11. Dimopoulos M., Kyle R., Fermand J.P. San Miguel J., Chanan-Khan A., Ludwig H., Joshua D., Mehta J., Gertz M., Avet-Loiseau H., Beksaç M., Anderson K.C., Moreau P., Singhal S., Goldschmidt H., Boccadoro M., Kumar S., Giralt S., Munshi N.C., Jagannath S. Consensus recommendations for standard investigative workup: report of the International Myeloma Workshop Consensus. Panel 3. Blood. 2011. vol. 117. no. 18. P. 4701-4705.

12. Haynes R.J., Read S., Collins G.P., Darby S.C., Winearls C.G. Presentation and survival of patients with severe acute kidney injury and multiple myeloma: a 20-year experience from a single centre. Nephrol Dial Transplant. 2010. no. 2. P. 419-426.

13. Gavriatopoulou M., Terpos E., Kastritis E., Dimopoulos M.A. Current treatments for renal failure due to multiple myeloma. Expert Opin Pharmacother. 2016. vol. 17. no. 16. P. 2165-2773.

14. Leung N., Nasr S.H. Myeloma-related kidney disease. Adv Chronic Kidney Dis. 2014. vol. 21. no. 1. P. 36-47.

15. Салогуб Г.Н. Осложнения множественной миеломы и методы их коррекции (лекция) // Вестник гематологии. 2014. Т.10. №3. С. 39-52.

16. Шеховцева Т.Г., Долинная М.А. Варианты поражения почек у больных 
множественной миеломой. Клиническое наблюдение: научное издание // Нефрология и диализ. 2014. Т. 16. № 3. С. 378-381.

17. Campos M.L., Barbosa-de Carvalho N.M., Martín-Reyes G. The value of serum free light chain assay in patients with monoclonal gammopathies and renal failure. Nefrologia. 2012. vol. 32. no. 1. P. 15-19.

18. Томилина Н.А., Подкорытова О.Л. Острая почечная недостаточность // Нефрология и диализ. 2009. Т. 11. №1. С. 4-20.

19. Faiman B.M., Mangan P., Spong J., Tariman J.D. Renal complications in multiple myeloma and related disorders: survivorship care plan of the International Myeloma Foundation Nurse Leadership Board. Clin. J. Oncol. Nurs. 2011. vol. 15. P. 66-76.

20. Рехтина И.Г., Марьина С.А., Тангиева Л.М., Калинин Н.Н., Бирюкова Л.С. Эффективность эксракорпоральных методов в элиминации легких цепей у больных множественной миеломой на программном гемодиализе // Гематология и трансфузиология. 2013. T.58. №2. C. 29-32.

21. Лучинин, А.С., Семочкин С.В., Минаева Н.В., Поздеев Н.М., Парамонов И.В. Эпидемиология множественной миеломы по данным анализа популяционного регистра Кировской области // Онкогематология. 2017. Т. 12. №3. С.50-56.

22. Скворцова, Н.В. Поспелова Т.И., Ковынев И.Б., Солдатова Г.С., Нечунаева И.Н. Эпидемиология множественной миеломы в Новосибирске (Сибирский федеральный округ) // Клиническая онкогематология. 2019. T.12, №1. C. 86-94. DOI: 10.21320/2500-2139-209-12-186-94.

23. Richardson P.G. Sonneveld P., Schuster M.W., Irwin D., Stadtmauer E.A., Facon T., Harousseau J.L., Ben-Yehuda D., Lonial S., Goldschmidt H., Reece D., San-Miguel J.F., Bladé J., Boccadoro M., Cavenagh J., Dalton W.S., Boral A.L., Esseltine D.L., Porter J.B., Schenkein D., Anderson K.C. Bortezomib or high-dose dexamethasone for relapsed multiple myeloma. N. Engl. J. Med. 2005. vol. 352. no. 24. P. 2487-2498.

24. Dimopoulos, M.A., Kastritis E., Rosinol L., Bladé J., Ludwig H. Pathogenesis and treatment of renal failure in multiple myeloma. Leukemia 2008. vol. 22. no. 8. P. 1485-1493.

25. Kyle R.A., Rajkumar S.V. Multiple myeloma. Blood 2008. vol. 111. no. 6. P. 2962-2972.

26. Хорошилов, С.Е., Никулин А.В. Детоксикация при критических состояниях: понимание научной проблемы в XXI веке (обзор) // Общая реаниматология. 2017. Т.13. №5. C.85-108.

27. Диагностика и лечение миеломной нефропатии. Клинические рекомендации. М., 2014. $17 \mathrm{c}$.

28. Finnish Medical Society Duodecim. Multiple myeloma (MM). In: EBM Guidelines. 
Evidence-Based Medicine. Helsinki, Finland: Wiley Interscience. John Wiley and Sons. 2007 May 30.

29. Smith A., Wisloff F., Samson D. Guidelines on the diagnosis and management of multiple myeloma 2005. Br. J. Haematol. 2006. vol. 132. no. 4. P. 410-451.

30. Бирюкова Л.С., Тангиева Л.М., Тимохов В.С., Фетисова Е.В., Пивник А.В., Марьина С.А. Комплексная терапия хронической почечной недостаточности у больных миеломной болезнью // Нефрология и диализ. 2002. Т. 4. № 2. С. 98-105.

31. Kyle R.A., Gertz M.A., Witzing T.E. Review of 1027 patients with newly diagnosed multiple myeloma. Mayo Clin. Proc. 2003. vol. 1. P. 21-33.

32. Hutchison C.A., Sanders P.W. Evolving strategies in the diagnosis, treatment, and monitoring of myeloma kidney. Adv Chronic Kidney Dis. 2012. vol. 19. no. 5. P. 279-281.

33. Finkel K.W., Cohen E.P., Shirali A. Paraprotein-related kidney disease: evaluation and treatment of myeloma cast nephropathy. Clin. J. Am. Soc. Nephrol. 2016. vol. 11. no. 12. P. 22732279.

34. Donati G., Moretti M.I., Baraldi O., Spazzoli A., Capelli I., Comai G., Marchetti A., Sarma M., Mancini R., La Manna G. Removal of free light chains in hemodialysis patients without multiple myeloma: a crossover comparison of three different dialyzers. BMC Nephrol. 2016. vol. 17. no. 1. P. 193.

35. Рехтина И.Г., Менделеева Л.П., Варламова Е.Ю., Бирюкова Л.С. Сравнение эффективности бортезомибсодержащих программ в достижении раннего гематологического и почечного ответа у больных миеломной нефропатией с диализзависимой почечной недостаточностью // Гематология и трансфузиология. 2015. Т.60. №4. С. 4-7.

36. Любимова Н.В., Тимофеев Ю.С., Громова Е.Г., Вотякова О.М., Огнерубова Н.А., Кушлинский Н.Е. Селективная элиминация свободных легких цепей иммуноглобулинов у больных моноклональными гаммапатиями // Вестник Тамбовского университета. Серия Естественные и технические науки. 2017. Т. 22. №1. С. 142-147.

37. Громова Е.Г., Зейналова П.А., Любимова Н.В., Тимофеев Ю.С., Н.Е., Семенова А.А., Лунин В.В., Ключагина Ю.И. Опыт селективной элиминации свободных легких цепей иммуноглобулинов у пациентов с моноклональными гаммапатиями // Онкогематология. 2019. T.14. №2. C. 8-12. 